\title{
Adaptive Full Scan Model for Range Finders in Dynamic Environments
}

Tinne De Laet, Joris De Schutter and Herman Bruyninckx

Department of Mechanical Engineering,

Katholieke Universiteit Leuven,

Celestijnenlaan 300B, B-3001 Leuven, Belgium.

Tinne.DeLaet@mech. kuleuven. be

http://people.mech.kuleuven.be/ tdelaet

Summary. Sensor models directly influence the efficiency and robustness of the estimation processes used in robot and object localization. Therefore this paper focusses on a probabilistic range finder sensor model for dynamic environments. The dynamic nature results from the presence of unmodeled and possibly moving objects and people.

The goal of this paper is twofold. First of al we present experiments to validate the Rigorously Bayesian Beam Model (RBBM), a new model we proposed in a previous paper. Second, we propose a sample-based full scan model to improve the state of the art models. In contrast to these Gaussian-based state of the art full scan models, the proposed model is able to handle the multi-modality of the range finder data, which is shown here to occur even in simple static environments.

\section{Introduction}

Intelligent robots require sensors to perceive their environment. To translate sensor measurements into intelligent behavior, the measurements have to be interpreted in the context of a physical sensor model. The design of a sensor model is a trade-off between accuracy (hence, increased intelligence) on the one hand, and simplicity and robustness on the other hand. While simplicity and robustness are improved by only using physically interpretable parameters, accuracy requires that: (i) the mathematical sensor model approximates the physical sensor well, and (ii) conditional probabilities on a well-chosen subset of the model parameters represent the stochastic nature of the involved physics.

In a probabilistic approach, inaccuracies are embedded in the stochastic nature of the model, particularly in the conditional probability density representing the measurement process. This way, good results and intelligent behavior are achieved using a limited modeling effort. Nevertheless, it is of vital importance that all types of inaccuracies affecting the measurements are 
incorporated in the probabilistic sensor model. Inaccuracies arise from sensor limitations, noise, and the fact that most complex environments can only be represented and perceived in a limited way. The dynamic nature of the environment in particular is an important source of inaccuracies. This dynamic nature results from the presence of unmodeled and possibly moving objects and people.

This paper focuses on probabilistic range finder sensor models for dynamic environments. Range finders, which are widely used in mobile robotics, measure the distances $\boldsymbol{z}$ to objects in the environment along certain directions $\boldsymbol{\theta}$ relative to the sensor. The sensor model is used in a form suitable for mobile robot localization, i.e.: $P(Z=z \mid X=x, M=m)^{1}$, where $Z$ indicates the measured range, $X$ the position of the mobile robot (and of the sensor mounted on it), and $M$ the environment map. The models are however useful in other applications of range sensors as well.

This paper is organized as follows. After discussing related work in the next section, Section 3 discusses the RBBM as it was proposed in [1] and presents new experimental results to validate this model. Section 4 presents the sample-based full scan model which is able to handle the multi-modality of the range finder data. Finally, Section 5 summarizes the results of the paper.

\section{Related Work}

The range finder sensor models available from literature are traditionally divided in three main groups: feature-based approaches, beam-based models and correlation-based methods. Feature-based approaches extract a set of features from the range scan and match them to features contained in an environmental model. Beam-based models, also known as ray cast models, consider each distance measurement along a beam as a separate range measurement. These models represent the one-dimensional distribution of the distance measurement by a parametric function, which depends on the expected range measurement in the respective beam directions. In addition, these models are closely linked to the geometry and the physics involved in the measurement process. They often result in overly peaked likelihood functions due to the underlying assumption of independent beams. The last group of range finder sensor models, correlation-based methods, build local maps from consecutive scans and correlate them with a global map. The simple and efficient likelihood field models or end point model [2] are related to these correlation-based methods. A nice summary of the advantages and drawbacks of the different range finder sensor models is given in [3].

\footnotetext{
${ }^{1}$ To simplify notation, the explicit mention of the random variable in the probabilities is omitted whenever possible, and replaced by the common abbreviation $P(x)$ instead of writing $P(X=x)$.
} 
Range finder sensor models can also be classified according to whether they use discrete geometric grids [4-8] or continuous geometric models [9-11]. Moravec [8] proposed non-Gaussian measurement densities over a discrete grid of possible distances measured by sonar; the likelihood of the measurements has to be computed for all possible positions of the mobile robot at a given time. Even simplified models [7] in this approach turned out to be computationally too expensive for real-time application. Therefore, Fox et al. proposed a beam model consisting of a mixture of two physical causes for a measurement: a hit with an object in the map, or with an object not yet modeled in the map [6]. The last cause accounts for the dynamic nature of the environment. An analogous mixture adds two more physical causes: a sensor failure and an unknown cause resulting in a 'max-range' measurement and a 'random' measurement, respectively $[9,10]$. While $[9,11]$ use a continuous model, $[10]$ presents the discrete analog of the mixture, taking into account the limited resolution of the range sensor. [11] extend the basic mixture model for use in Monte Carlo localization. To overcome problems due to the combination of the limited representational power and the peaked likelihood of the accurate range finder, they propose an adaptive likelihood model. The likelihood model is smooth during global localization and more peaked during tracking.

Recently, different papers tried to tackle the problems associated with beam-based models, caused by the independence assumptions between beams. [3] propose a sensor model for the full scan. The model treats the sensor modeling task as a non-parametric Bayesian regression problem, and solves it using Gaussian processes. It is claimed that the Gaussian beam processes combine the advantages of the beam-based and the correlation-based models. Due to the underlying assumption that the measurements are jointly Gaussian distributed, the Gaussian beam processes are not suited to take into account the non-Gaussian uncertainty due to the dynamic nature of the environment. An alternative approach to handle the overly-peaked likelihood functions resulting from the traditional beam models is proposed in [12]. A location-dependent full scan model takes into account the approximation error of the sample-based representation, and explicitly models the correlations between individual beams introduced by the pose uncertainty. Despite the modeled correlation between beams, the measurements are still assumed to be jointly Gaussian distributed, which again limits the applicability in dynamic environments. Pfaff et al. [11] extend the basic mixture model for use in Monte Carlo localization. To overcome problems due to the combination of the limited representational power and the peaked likelihood of the accurate range finder, they propose an adaptive likelihood model. The likelihood model is smooth during global localization and more peaked during tracking.

Recently, different papers tried to tackle the problems associated with beam-based models, caused by the independence assumptions between beams. [3] propose a sensor model for the full scan. The model treats the sensor modeling task as a non-parametric Bayesian regression problem, and solves it using Gaussian processes. It is claimed that the Gaussian beam processes combine 
the advantages of the beam-based and the correlation-based models. Due to the underlying assumption that the measurements are jointly Gaussian distributed, the Gaussian beam processes are not suited to take into account the non-Gaussian uncertainty due to the dynamic nature of the environment. An alternative approach to handle the overly-peaked likelihood functions resulting from the traditional beam models is proposed by Pfaff et al. [12]. A location-dependent full scan model takes into account the approximation error of the sample-based representation, and explicitly models the correlations between individual beams introduced by the pose uncertainty. Despite the modeled correlation between beams, the measurements are still assumed to be jointly Gaussian distributed, which again limits the applicability in dynamic environments.

\section{Rigorously Bayesian Beam Model}

This section gives first a brief description of the RBBM and the differences with respect to the state of the art models. Next, additional experiments are presented that show that the RBBM has the same representational power as the state of the art models.

\subsection{Description}

We recently proposed a rigorously Bayesian modeling of the probabilistic range sensor beam model for dynamic environments, referred to as RBBM [1]. Similar to $[9,11]$ the sensor model is derived for a continuous geometry. Unlike previous models $[6,9-11]$, the mixture components are founded on a Bayesian modeling. This modeling makes use of probabilistic graphical models, in this case Bayesian networks.

The obtained RBBM can be written as a mixture of four possible causes of a range measurement: a hit with the map, a hit with an unmodeled object, an unknown cause resulting in a random measurement and a sensor failure resulting in a maximum reading measurement:

$$
\begin{aligned}
P(z \mid x, m)=\pi_{1} P_{\text {hit }}(z \mid x, m)+ & \pi_{2} P_{\text {occl }}(z \mid x, m)+ \\
& \pi_{3} P_{\text {rand }}(z \mid x, m)+\pi_{4} P_{\max }(z \mid x, m),
\end{aligned}
$$

where, 


$$
\begin{aligned}
\pi_{1} & =\left(1-p^{\prime}\right)\left(1-\pi_{3}-\pi_{4}\right), \\
\pi_{2} & =p^{\prime}\left(1-\pi_{3}-\pi_{4}\right), \\
P_{\text {hit }}(z \mid x, m) & =\mathcal{N}\left(z ; z^{\star}, \sigma_{m}\right), \\
P_{\text {occl }}(z \mid x, m) & =\left\{\begin{array}{ll}
\frac{1}{z^{\star}} \frac{1-p^{\prime}}{\left[1-\left(\frac{z^{\star}-z}{z^{\star}} p^{\prime}\right)\right]^{2}} & \text { if } 0 \leq z \leq z^{\star} \\
0 & \text { otherwise. }
\end{array},\right. \\
P_{\text {rand }}(z \mid x, m) & =\left\{\begin{array}{ll}
\frac{1}{z_{\max }} & \text { if } 0 \leq z \leq z_{\max }, \\
0 & \text { otherwise. }
\end{array},\right. \text { and } \\
P_{\max }(z \mid x, m) & =I\left(z_{\max }\right)= \begin{cases}1 & \text { if } z=z_{\max }, \\
0 & \text { otherwise. }\end{cases}
\end{aligned}
$$

with $z^{\star}$ is the distance the range sensor would ideally measure, i.e. the distance to the closest object in the map.

The RBBM, Eq. (1-7), depends on four independent model parameters:

$$
\Theta=\left[\sigma_{m}, p^{\prime}, \pi_{3}, \pi_{4}\right],
$$

while $z_{\max }$ is a known sensor characteristic. This set of parameters has a clear physical interpretation; $\sigma_{m}$ is the standard deviation of the zero mean Gaussian measurement noise governing $P_{\text {hit }}(z \mid x, m) ; p^{\prime}$ is the probability that the map is occluded; $\pi_{3}$ and $\pi_{4}$ are the probabilities that the range finder returns an unexplainable measurement (unknown cause) and a maximum reading (sensor failure), respectively. We presented a maximum-likelihood (ML) estimation and a variational Bayesian (VB) estimation algorithm both based on the expectation-maximization approach to learn the RBBM model parameters [1].

With respect to the state of the art beam model of Thrun et al. [9], the RBBM has: (i) a different functional form for the probability of range measurements caused by unmodeled objects, (ii) an intuitive explanation for the discontinuity encountered in the cited paper, and (iii) a reduction in the number of model parameters. The presented ML and VB estimation algorithms directly benefit from the reduced number of model parameters.

\subsection{Experiment}

In the learning experiment, the experimental data reported by Thrun et al. [9] is used. The data consists of two series of measurements obtained with a mobile robot traveling through a typical office environment. From the set of measurements, 10000 measurements that are centered around two different expected ranges, are selected. The two obtained sets with different expected ranges are shown in Fig. 1.

The goal of this section is threefold: (i) to learn the model parameters (8) of the RBBM (1) from experimental data, (ii) to compare the results of 


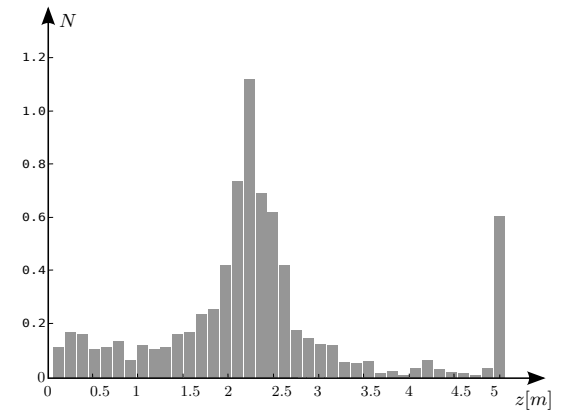

(a) Short range

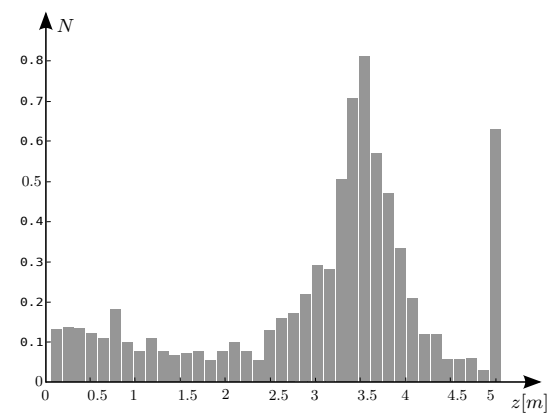

(b) Long range

Fig. 1. Data for second learning experiment reported in [9]. These data consist of two series of measurements obtained with a mobile robot traveling through a typical office environment. From the set of measurements 10000 measurements that are centered around two different expected ranges are selected.

the ML-EM and VB-EM estimator, and (iii) to compare the results of the proposed estimators with the learning approach of Thrun's model [9].

To see how well the learned model explains the experiment, the learned continuous pdf $P(z \mid x, m, \Theta)$ of Eq. (1) has to be compared with the discrete pdf of the experimental data (histogram) $H(z)$. To this end, the learned pdf is first discretized using the same bins $\left\{z_{f}\right\}_{f=1: F}$ as the experimental pdf. To quantize the difference between the learned and the experimental pdf two 'distance' measures are used: the discrete KL-divergence:

$$
d_{1} \approx K L(H \| P) \approx \sum_{f=1}^{F} H\left(z_{l}\right) \log \frac{H\left(z_{f}\right)}{P\left(z_{f} \mid x, m, \Theta\right)},
$$

and the square root of the discrete Hellinger distance:

$$
d_{2} \approx D H(H \| P) \approx \sqrt{\sum_{f=1}^{F}\left(H\left(z_{f}\right)^{\frac{1}{2}}-P\left(z_{f} \mid x, m, \Theta\right)^{\frac{1}{2}}\right)^{2}} .
$$

The latter is known to be a valid symmetric distance metric [13].

The parameters of the learning algorithms are listed in Table 1. Fig. 2 and Table 2 show the results of the ML-EM and VB-EM estimators for the RBBM compared to the results of the ML estimator for Thrun's model [9] for these two sets. The results are obtained by running the learning algorithms for 30 iteration steps. The proposed ML-EM and VB-EM estimator outperform the ML-EM estimator for Thrun's model [9] for the studied data sets. Despite the reduced number of parameters of the RBBM compared to Thrun's model, the RBBM has at least the same representational power. 


\begin{tabular}{c|c|c} 
ML-EM RBBM & VB-EM RBBM & ML-EM Thrun's model \\
\hline$\sigma_{m, \text { init }}=0.5$ & $p_{\text {init }}^{\prime}=\frac{1}{3}$ & $\sigma_{m, \text { init }}=0.5$ \\
$p_{\text {init }}^{\prime}=0.4$ & $\beta_{\text {init }}=5000$ & $z_{\text {hit,init }}=0.4$ \\
$\pi_{3, \text { init }}=0.2$ & $W_{\text {init }}=12$ & $z_{\text {short }, \text { init }}=0.3$ \\
$\pi_{4, \text { init }}=0.1$ & $\bar{\mu}_{\text {init }}=x_{m p}$ & $z_{\text {max }, \text { init }}=0.1$ \\
& $\nu_{\text {init }}=100$ & $z_{\text {rand,init }}=0.2$ \\
& $\alpha_{1, \text { init }}=\frac{5}{8}$ & $\lambda_{\text {short }, \text { init }}=14$ \\
& $\alpha_{2, \text { init }}=\frac{1}{8}$ & \\
& $\alpha_{3, \text { init }}=\frac{1}{8}$ & \\
& $\alpha_{4, \text { init }}=\frac{1}{8}$ & \\
& $\beta_{0}=5$ & \\
& $W_{0}=50$ & \\
& $\bar{\mu}_{0}=x_{m p}$ & \\
& $\nu_{0}=100$ & \\
& $\alpha_{0}=1$ &
\end{tabular}

Table 1. EM-parameters for the learning experiment (all in SI-units). In the ML approaches, the mean of $P_{\text {hit }}(z \mid x, m)$ is set to $x_{m p}$, i.e. the most probable bin of the histogram of the training set $H(z)$

(a) Discrete KL-divergence

\begin{tabular}{c|ccc} 
Experiment & \multicolumn{3}{|c}{$\boldsymbol{d}_{\mathbf{1}}$ (Eq. (9)) } \\
\hline \hline & ML-EM & VB-EM & ML-EM \\
& RBBM & RBBM & Thrun's model \\
\hline short range & 0.5295 & 0.5127 & 0.7079 \\
long range & 0.4366 & 0.4368 & 0.5852 \\
\hline average & 0.4830 & 0.4747 & 0.6465
\end{tabular}

(b) square root Hellinger distance

\begin{tabular}{c|ccc} 
Experiment & \multicolumn{3}{|c}{$\boldsymbol{d}_{\mathbf{2}}$ (Eq. (10)) } \\
\hline \hline & ML-EM & VB-EM & ML-EM \\
RBBM & RBBM & Thrun's model \\
\hline short range & 0.3166 & 0.2971 & 0.5629 \\
long range & 0.1683 & 0.2100 & 0.3481 \\
\hline average & 0.2425 & 0.2535 & 0.4555
\end{tabular}

Table 2. Discrete KL-divergence and square root Hellinger distance for the second learning experiment between the training set and the results of the ML-EM and VB-EM estimators for the RBBM and the ML-EM estimator for Thrun's model [9] 


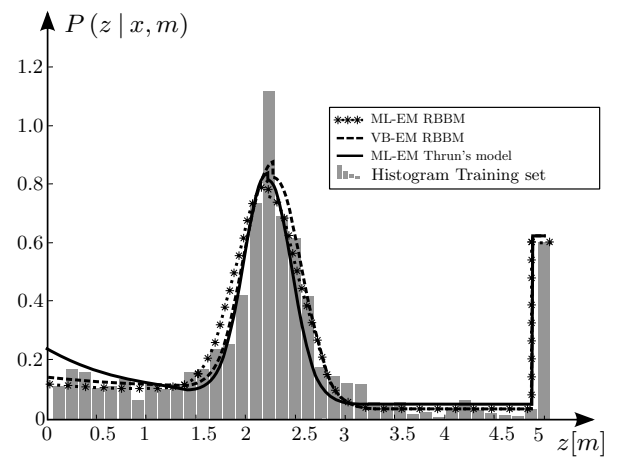

(a) Short range

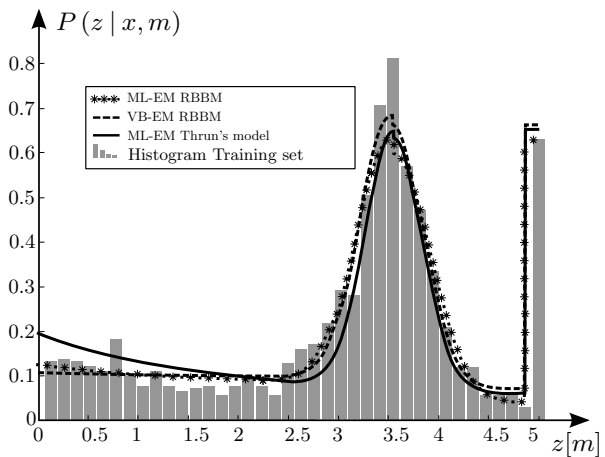

(b) Long range

Fig. 2. Comparison of the results of the ML-EM and VB-EM estimators for the RBBM and the results of a maximum likelihood estimator for Thrun's model [9] for the data of Fig. 1.

\section{Sample-based Full Scan Model}

This section extends the RBBM (1)to an adaptive full scan model for dynamic environments; adaptive, since it automatically adapts to the local density of samples when using sample-based representations; full scan, since the model takes into account the dependencies between individual beams.

In many applications using a range finder, the posterior is approximated by a finite set of samples (histogram filter, particle filters). The peaked likelihood function associated with a range finder (small $\sigma_{m}$ due to its accuracy) is problematic when using such finite set of samples. The likelihood $P(z \mid x, m)$ is evaluated at all samples, which are approximately distributed according to the posterior estimate. Basic sensor models typically assume that the estimate $x$ and the map $m$ are known exactly, that is, they assume that one of the samples corresponds to the true value. This assumption, however, is only valid in the limit of infinitely many samples. Otherwise, the probability that a value exactly corresponds to the true location is virtually zero. As a consequence, these peaked likelihood functions do not adequately model the uncertainty due to the finite, sample-based representation of the posterior [12]. Furthermore, the use of a basic range finder model typically results in even more peaked likelihood models, especially when using a large number of beams per measurement, due to multiplication of probabilities. In practice, the problem of peaked likelihoods, is dealt with in various ways: sub-sampling the measurement (fewer beams); introducing minimal likelihoods for beams; inflating the measurement uncertainty; or other means of regularization of the resulting likelihoods. These solutions are not satisfactory however, since the additional uncertainty due to the sample-based representation is not known in advance. The additional uncertainty strongly varies with the number of samples and 
the uncertainty of the estimate [11]. Fox et al. [14] propose to dynamically adapt the number of samples by means of KLD sampling. For very peaked likelihoods however, this might result in a huge number of samples. A possible solution is to ensure that a critical mass of samples is located at the important parts of the state space by sampling from the observation model $[3,12]$. Sampling from the observation model however, is often only possible in an approximate and inaccurate way. Pfaff et al. [11] introduced an adaptive beam model for dynamic environments, which explicitly takes location uncertainty due to the sample-based representation into account. They compute the additional uncertainty due to the sample-based representation, using techniques from density estimation. When evaluating the likelihood function at a sample, they consider a certain region around the sample, depending on the sample density at that location. Then, depending on the area covered by the sample, the variance of the Gaussian, $\sigma_{m}$, governing the beam model in Eq. (1), is calculated for each sample. As a result, the beam model automatically adapts to the local density of samples. Such a location dependent model results in a smooth likelihood function during global localization and a more peaked function during position tracking without changing the number of samples.

By considering a region around samples, the individual beams become statistically dependent $[3,12]$. The degree of dependency depends on the geometry of the environment and on the size and location of the considered region. Beam models, such as the RBBM, implicitly assume however that the beams are independent, that is:

$$
P(\boldsymbol{z} \mid \boldsymbol{\theta}, x, m)=\prod_{b=1}^{B} P\left(z_{b} \mid \theta_{b}, x, m\right),
$$

where $\boldsymbol{z}=\left\{z_{b}\right\}_{b=1: B}$ and $\boldsymbol{\theta}=\left\{\theta_{b}\right\}_{b=1: B}$ are the vectors containing the measured ranges and the angles of the different beams respectively; $z_{b}$ is the range measured at the beam with angle $\theta_{b} ; B$ is the total number of beams and $P\left(z_{b} \mid \theta_{b}, x, m\right)$ is for instance the RBBM (Eq. (1)). By neglecting the dependency between beams, the resulting likelihoods $P(\boldsymbol{z} \mid \boldsymbol{\theta}, x, m)$ are overly peaked. Models taking into account the dependencies between beams consider the full range scan and are therefore called full scan models further on. The previously proposed full scan models $[3,12]$ both assume that the beams of a range scan are jointly Gaussian distributed. The off-diagonal elements of the covariance matrix associated with the Gaussian distribution represent the dependency. To learn the model parameters, both methods draw samples from the region around a sample and perform ray-casting using these samples. Plagemann et al. [3] train a Gaussian process which models the full scan, while Pfaff et al. [12] directly provide a maximum likelihood estimate for the mean and covariance of the Gaussian.

Section 4.1 shows that the dependency between beams may introduce multi-modality, even for simple static environments. The multi-variate Gaussian models $[3,12]$ cannot handle this multi-modality. Therefore, a new sample- 
based method for obtaining an adaptive full scan model from a beam model, able to handle multi-modality, is proposed. Section 4.2 extends the adaptive full scan model for dynamic environments by taking into account nonGaussian model uncertainty.

\subsection{Sample-based Adaptive Full Scan Model for Static Environments}

Plagemann et al. [3] and Pfaff et al. [12] estimate the full scan model, $P(\boldsymbol{z} \mid x, m)^{2}$, based on a local environment $\mathcal{U}(x)$ of the exact estimate $x$ :

$$
P(\boldsymbol{z} \mid x, m) \approx \int P(\tilde{x} \mid x) P_{\mathrm{hit}}(\boldsymbol{z} \mid \tilde{x}, m) d \tilde{x},
$$

with $P(\tilde{x} \mid x)$ the distribution representing the probability that $\tilde{x}$ is an element of the environment $\mathcal{U}(x)$, i.e: $\tilde{x} \in \mathcal{U}(x)$. Since this section does not consider the dynamics of the environment, only one component of the RBBM in Eq. (1) is used: $P_{\text {hit }}(z \mid x, m)$. The marginalization over the environment $\mathcal{U}(x)$ in Eq.(12) introduces dependencies between the measurements $z_{b}$ of the measurement vector $\boldsymbol{z}$.

The environment $\mathcal{U}(x)$, as explained above, depends on the sample density around the sample $x$ under consideration. Pfaff et al. [11] propose to use a circular region with diameter $d_{\mathcal{U}(x)}$, which is a weighted sum of the Euclidean distance and the angular difference. Like Plagemann et al. [3] and Pfaff et al. [12], an approximation of the above likelihood can be estimated online for each sample $x$ by simulating $L$ complete range scans at locations drawn from $\mathcal{U}(x)$ using the given map $m$ of the environment. Contrary to the multivariate Gaussian approximation proposed in $[3,12]$, we propose a sample-based approximation, able to handle multi-modality. Sampling from the environment $\mathcal{U}(x)$ immediately results in a sample-based approximation of $P(\tilde{x} \mid x)$ :

$$
P(\tilde{x} \mid x) \approx \frac{1}{L} \sum_{l=1}^{L} \delta_{\tilde{x}^{(l)}},
$$

where $\delta_{\tilde{x}^{(l)}}$ denotes the delta-Dirac mass located in $\tilde{x}^{(l)}$, and the samples are distributed according to $P(\tilde{x} \mid x)$ :

$$
\tilde{x}^{(l)} \sim P(\tilde{x} \mid x) .
$$

Using this sample-based approximation of $P(\tilde{x} \mid x)$ the likelihood of Eq. (12) can be approximated as:

$$
P(\boldsymbol{z} \mid x, m) \approx \frac{1}{L} \sum_{l=1}^{L} P_{\text {hit }}\left(\boldsymbol{z} \mid \tilde{x}^{(l)}, m\right) .
$$

\footnotetext{
${ }^{2}$ To simplify the notation $\boldsymbol{\theta}$ and $\theta$ are omitted from $P(\boldsymbol{z} \mid \boldsymbol{\theta}, x, m)$ and $P\left(z_{b} \mid \theta_{b}, x, m\right)$, respectively.
} 
Since this sample-based approximation has to be calculated online, the number of samples has to be limited. If the used environment $\mathcal{U}(x)$ is large, the resulting approximation will be bad. To smooth the undesired bumpy behavior due to the limited number of samples, the measurement noise $\sigma_{m}$ governing $P_{\text {hit }}(z \mid x, m)$ in Eq. (4), is artificially increased depending on the size of $\mathcal{U}(x)$ by multiplying it with a factor:

$$
1+C \sqrt{d_{u(x)}} \text {. }
$$

In further simulations, $C$ was set to 20 .

\section{Experiment}

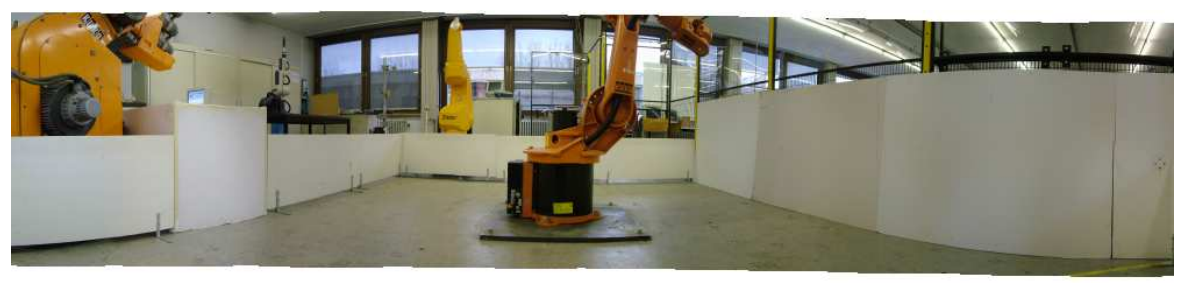

Fig. 3. Panorama taken from the Sick LMS 200 range finder mounted on the Kuka 361 industrial robot. The environment consists of a rectangular room with an object (a Kuka KR 15/2 robot) in the middle. We show that even for this simple static environment, the presented sample-based full scan model outperforms the Gaussianbased state of the art models.

A simple environment consisting of a rectangular room with an object (a Kuka KR 15/2 robot) in the middle (Fig. 3) is used to show that the marginalization over (even small) $\mathcal{U}(x)$ to obtain the true likelihood not only introduces dependencies between the beams but also multi-modality. The $\mathcal{U}(x)$ results from a local uncertainty on the $x$ - and $y$-position of $0.01 \mathrm{~m}$ and a rotational uncertainty of $5^{\circ}$. To obtain a reference, a Sick LMS 200 range finder is used to take a large number of measurements $(L=1500)$ at random locations sampled in $\mathcal{U}(x)$. To allow for exact positioning, the Sick LMS 200 is placed on a Kuka 361 industrial robot. The Sick LMS 200 range finder is connected to a laptop, communicating with the Kuka 361 industrial robot over the network using Corba-facilities in the Open Robot Control Software, Orocos ( $[15,16])$. A simplified map of the environment (Fig. 3) is built to simulate the 150 complete range scans needed to construct a full scan model. The marginal $P\left(z_{b} \mid x, m\right)$ of a selected beam (the middle one) is studied here in more detail. The marginal likelihoods for the selected beam using the proposed samplebased approximation of Eq. (15) and the Gaussian approximation proposed 
by [12], are compared in Fig. 4(a). The histogram of the measurements of the selected beam in this figure clearly shows the multi-modality of the likelihood caused by the dependency between beams. Fig. 4(d) shows the difference for all beams between the experimentally obtained cumulative marginal ( $L=1500)$ and the Gaussian-based and sample-based approximation for all beams. The mean difference with the experimental data for the sample-based approximation is 2.8 times smaller than the difference for the Gaussian-based approximation, even for the simple static environment of Fig. 3

\subsection{Sample-based Adaptive Full Scan Model for Dynamic Environments}

The adaptive beam model proposed in [11] is suited for use in dynamic environments since it uses the four component mixture beam model $[9,10]$. To date however, the available adaptive full scan likelihood models of [3,12] have not been adapted for dynamic environments. The assumption that the beams are jointly Gaussian distributed, unable to capture the non-Gaussian uncertainty due to environment dynamics, prevents the straightforward extension for dynamic environments. In contrast, the sample-based approximation of the full scan likelihood, as proposed in Section 4.1, can be extended to include environment dynamics. To this end, replace $P_{\text {hit }}(z \mid x, m)$ in Eq. (12) and Eq. (15) by the full mixture of Eq. (1).

\section{Experiment}

Fig. 5(a) and Fig. 5(b) compare the marginals for the selected beams (Fig. 4(a)) obtained from the adaptive full scan model for dynamic environments using the proposed sample-based approximation and the Gaussian approximation proposed by [12]. Fig. 5(c) shows a probability map of the adaptive full scan model suited for dynamic environments for the example environment of Fig. 3. The probability map shows that the marginalization over the environment $\mathcal{U}(x)$ of a sample in Eq. (12) not only introduces dependency between beams but also introduces multi-modality.

\section{Conclusion}

The contribution of this paper is twofold. First of al we presented experiments to validate the Rigorously Bayesian Beam Model (RBBM) [?]. The experiments showed that despite the reduced number of parameters of the RBBM compared to state of the art model of Thrun et al. [9], the RBBM has at least the same representational power.

Second, we proposed a sample-based full scan model to improve the state of the art models. In constrast to the multi-variate Gaussian state of the art 


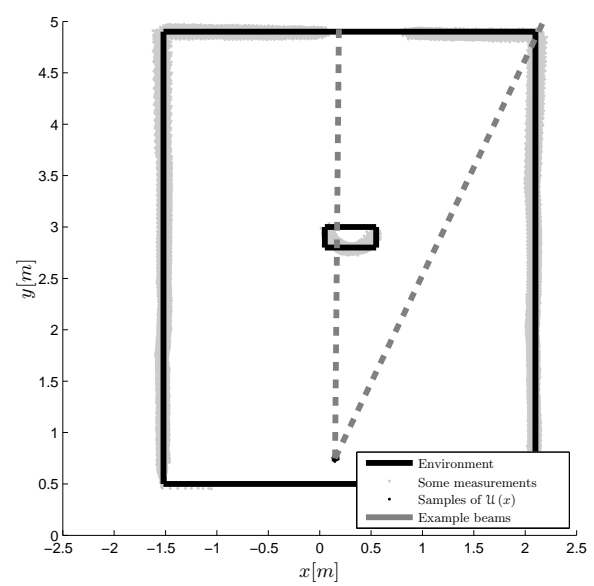

(a) Environment model

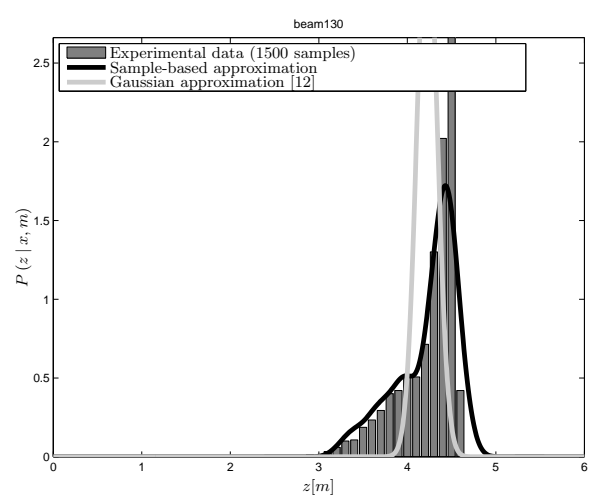

(c) Marginal likelihood for right beam

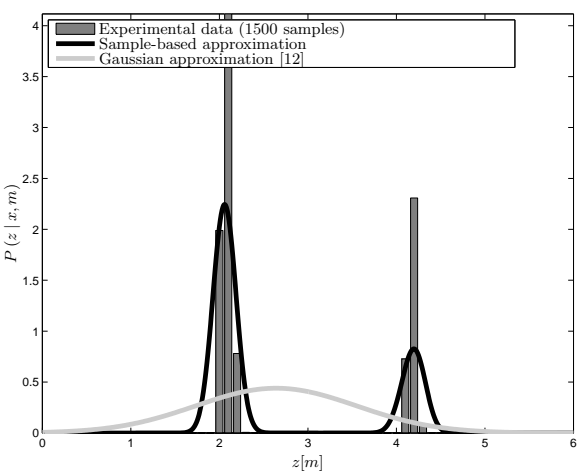

(b) Marginal likelihood for left beam

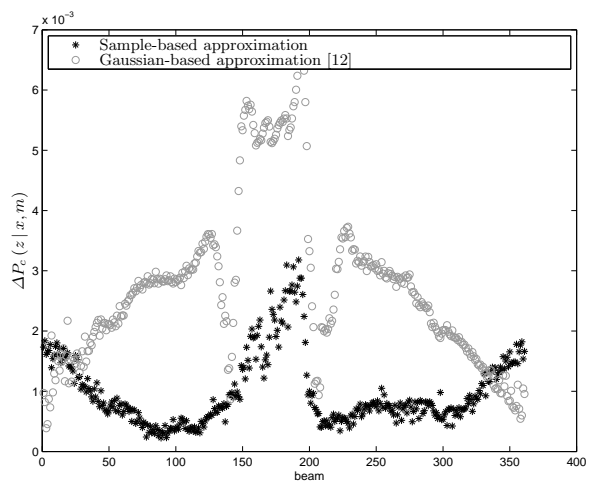

(d) Difference between experimental cumulative marginal and Gaussian and sample-based approximations

Fig. 4. Figure (a) indicates the model of the simple evnrionment of Fig. 3, consisting of a room with an object in the middle, with black lines. The range finder is located at $(0.15 m, 0.75 m)$. Samples from $\mathcal{U}(x)$ (resulting from a local uncertainty on the $x$ and $y$-position of $0.01 \mathrm{~m}$ and a rotational uncertainty of $5^{\circ}$ ) are shown with black dots, and some simulated measurements are shown in grey. Figure (b) and Figure(c) show the marginal likelihood $P\left(z_{b} \mid x, m\right)$ for a the two selected beams together with the histogram of the experimentally recorded range finder data, the Gaussian-based approximation $(L=150)$ of [12] and the sample-based approximation $(L=150)$. In contrast to the Gaussian-based state of the art full scan model, the proposed sample-based approximation is able to handle the multi-modality of the range finder data. Figure (d) shows the difference for all beams between the experimentally obtained cumulative marginal $(L=1500)$ and the Gaussian-based and sample-based approximation. The mean difference with the experimental data for the samplebased approximation is 2.8 times smaller than the difference for the Gaussian-based approximation, even for the simple static environment of Fig. 3 and the small $\mathcal{U}(x)$. 


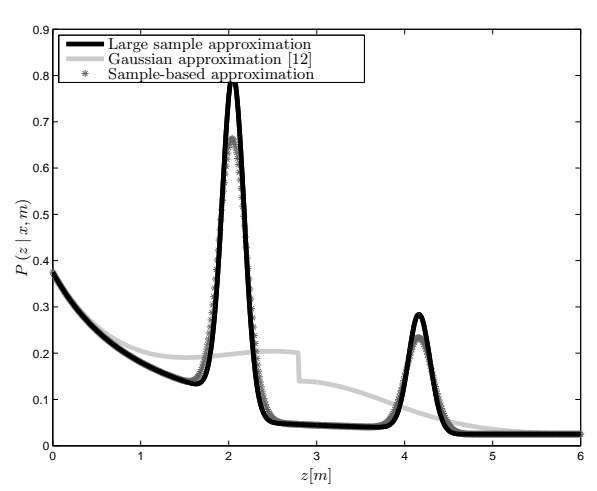

(a) Marginal likelihood for left beam

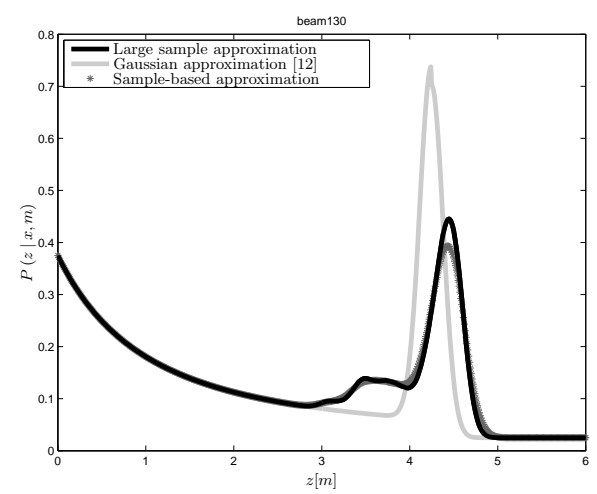

(b) Marginal likelihood for right beam

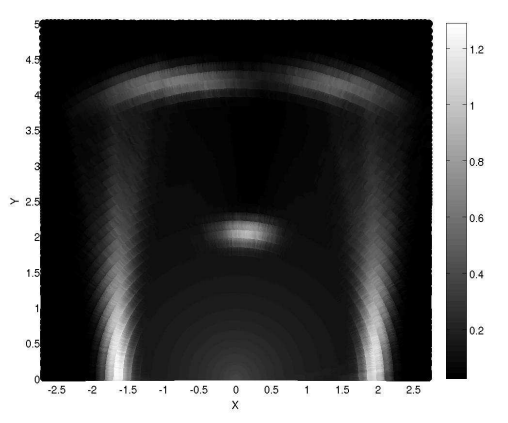

(c) Probability map

Fig. 5. Figure (a) and Figure(b) show the marginal likelihood $P\left(z_{b} \mid x, m\right)$ for a the two selected beams 4(a) together with the Gaussian-based approximation ( $L=150)$ of [12] and the sample-based approximation $(L=150)$ extended for the use in dynamic environments. In contrast to the Gaussian-based state of the art full scan model, the proposed sample-based approximation is able to handle the multimodality of the range finder data. Figure (c) shows the probability map for a large number of beams.

models, the proposed sample-based method is able to handle multi-modality. This multi-modality was shown to occur even in simple static environments. The sample-based approximation was shown to better explain experimentally recorded range finder data. The mean difference with the experimental data for the sample-based approximation is 2.8 times smaller than the difference for the Gaussian-based approximation. The proposed adaptive full scan model was extended for use in dynamic environments by taking into account non-Gaussian model uncertainty. To this end, the Rigorously Bayesian Beam Model (RBBM) was extended to a full scan model. 


\section{Acknowledgment}

All authors gratefully acknowledge the financial support by K.U.Leuven's Concerted Research Action GOA/05/10 and the Research Council K.U.Leuven, $\mathrm{CoE}$ EF/05/006 Optimization in Engineering (OPTEC). Tinne De Laet is a Doctoral Fellow of the Fund for Scientific Research-Flanders (F.W.O.) in Belgium.

\section{References}

1. T. De Laet, J. De Schutter, and H. Bruyninckx, "Rigorously Bayesian range finder sensor model for dynamic environments," in Proceedings of the 2008 IEEE International Conference on Robotics and Automation, Pasadena, California, U.S.A., 2008.

2. S. Thrun, "A probabilistic online mapping algorithm for teams of mobile robots," The International Journal of Robotics Research, vol. 20, no. 5, pp. 335-363, 2001.

3. C. Plagemann, K. Kersting, P. Pfaff, and W. Burgard, "Gaussian beam processes: A nonparametric bayesian measurement model for range finders," in Robotics: Science and Systems (RSS), Atlanta, Georgia, USA, June 2007.

4. D. Hähnel, D. Schulz, and W. Burgard, "Mobile robot mapping in populated environments and sensor planning," Journal of the Advanced Robotics, vol. 17, no. 7 , pp. 579-597, 2003.

5. D. Hähnel, R. Triebel, W. Burgard, and S. Thrun, "Map building with mobile robots in dynamic environments," in Proceedings of the 2003 IEEE International Conference on Robotics and Automation. Taipeh, Taiwan: ICRA2003, 2003, pp. $1557-1569$.

6. D. Fox, W. Burgard, and S. Thrun, "Markov localization for mobile robots in dynamic environments," Journal of Artificial Intelligence Research, vol. 11, pp. 391-427, 1999.

7. W. Burgard, D. Fox, D. Hennig, and T. Schmidt, "Estimating the absolute position of a mobile robot using position probability grids," in Proc. of the National Conference on Artificial Intelligence, 1996.

8. H. P. Moravec, "Sensor fusion in certainty grids for mobile robots," AI Magazine, vol. 9, pp. 61-74, 1988.

9. S. Thrun, W. Burgard, and D. Fox, Probabilistic Robotics. MIT Press, 2005.

10. H. Choset, K. M. Lynch, S. Hutchinson, G. A. Kantor, W. Burgard, L. E. Kavraki, and S. Thrun, Principles of Robot Motion: Theory, Algorithms, and Implementations. MIT Press, June 2005.

11. P. Pfaff, W. Burgard, and D. Fox, "Robust monte-carlo localization using adaptive likelihood models," in European Robotics Symposium, H. Christensen, Ed., vol. 22. Palermo, Italy: Springer-Verlag Berlin Heidelberg, Germany, March 2006, pp. 181-194.

12. P. Pfaff, C. Plagemann, and W. Burgard, "Improved likelihood models for probabilistic localization based on range scans," in Proceedings of the 2007 IEEE/RSJ International Conference on Intelligent Robots and Systems. San Diego, California: IROS2007, 2007. 
13. C. M. Bishop, Pattern Recognition and Machine Learning. Springer, 2006.

14. D. Fox, "Adapting the Sample Size in Particle Filters Through KLD-Sampling," The International Journal of Robotics Research, vol. 22, no. 12, pp. 985-1003, December 2003.

15. H. Bruyninckx, "Open RObot COntrol Software," http://www.orocos.org/, 2001.

16. P. Soetens, "A software framework for real-time and distributed robot and machine control," Ph.D. dissertation, Department of Mechanical Engineering, Katholieke Universiteit Leuven, Belgium, May 2006. 